\title{
Bifurcating DNS Thresholds in a Model of Organizational Bridge Building *
}

\author{
Jonathan P. Caulkins ${ }^{\dagger} \quad$ Gustav Feichtinger ${ }^{\ddagger} \quad$ Dieter Grass $^{\ddagger \S}$ \\ and Gernot Tragler
}

\begin{abstract}
A simple optimal control model is introduced, where "bridge building" positions are rewarded. The optimal solutions can be classified in regards of the two extern parameters, (1) costs for the control staying at such an exposed position and (2) the discount rate. A complete analytical description of the bifurcation lines in parameter space is derived, which separates regions with different optimal behavior. These are resisting the influence from inner and outer forces, always fall off from the boundaries or decide based on one's initial state. This latter case gives rise to the emergence of so-called Dechert-Nishimura-Skiba (DNS) points describing optimal solution strategies. Furthermore the bifurcation from a single DNS point into two DNS points has been analyzed in parameter space. All these strategies have a funded interpretation within the limits of the model.
\end{abstract}

Key Words: Optimal control, thresholds, multiple equilibria, political behavior

\footnotetext{
*This research was partly financed by the Austrian National Bank (ÖNB) under grant No. 9414.

We thank David Krackhardt and Vladimir Veliov for many helpful comments and suggestions. ${ }^{\dagger}$ H. John Heinz III School of Public Policy and Management, Carnegie Mellon University, 5000 Forbes Ave., Pittsburgh, PA 15213, U.S.A.

$\ddagger$ Department of Operations Research and Systems Theory, Vienna University of Technology, Argentinierstrasse 8, A-1040 Vienna, Austria

$\S$ Corresponding Author
} 


\section{Introduction}

Connectors that tie together disparate objects are often under stress, but they are crucially important, whether the objects are physical or social. Welding joints and metal fasteners are common failure points in mechanical structures, and they are appropriately the focus of design effort. Social networks can be similar.

People who straddle two groups or organizations may be pulled in competing directions, but they can also exploit their position to control information flows and create value. E. g., they can market their home organization to the outside world and serve as a conduit for ideas and information flowing into the organization from the outside. It takes effort to maintain connections with diverse audiences; it is usually easier to establish relationships with people who are similar and to maintain relationships with people one sees routinely than to do so with outsiders. Yet people who manage to be bridge builders are rewarded for their special position.

Social network researchers have found that being "in the middle" of an organization (known as "centrality") confers advantages, including power (Refs. Krackhardt, 1990; Brass and Burkhardt, 1992). They distinguish among (at least) three types of centrality (Ref. Freeman, 1979). "Degree centrality" refers to the number of people to whom one is connected. "Closeness" is self-explanatory; individuals connected to many others by relatively direct paths, with few intermediaries, score high on closeness measures. "Betweenness" centrality refers to the extent to which an actor falls between pairs of other actors on the shortest 
paths (geodesics) connecting them.

The focus here is on betweenness. Its value is intuitive. If one person is the sole connection between two others, that intermediary has unique bargaining power with respect to any beneficial exchanges among those so connected. In effect, he or she has a monopoly over brokerage services between those powers. When the intermediary connects not just individuals but distinct groups each with multiple internal connections but with no overlap between groups the bridging person benefits all the more.

This idea is at least as old as Medieval Venice profiting by connecting Western Europe with the Orient, but in modern social network theory it is closely associated with Mark Granovetter's classic (Ref. Granovetter, 1973) article on "The Strength of Weak Ties." Subsequent authors e.g., (Ref. Burt, 1992) have argued that the key is not that the ties are weak, but that they be nonredundant "information bridges" that overcome "structural holes" in the organizational network. "Information benefits are expected to travel over all bridges, strong or weak. ... The task for a strategic player building an efficient-effective network is to focus resources on the maintenance of bridge ties." (Ref. Burt, 1992, p. 75)

This paper introduces a very simple model that reflects the challenges and benefits of building bridges by standing at the edge of one's home organization and reaching out to the external world. It describes when various professional strategies are preferred as a function of one's level of patience (discount rate) and the cost of adjusting one's social position. For many sets of parameter values, the solution is characterized by so-called Dechert-Nishimura-Skiba (DNS) points 
(Refs. Dechert and Nishimura, 1983; Skiba, 1978). At such points the decision maker is indifferent which policy to choose as there exist at least two optimal policies which are equally optimal. In particular, as the rate of time preference varies, a single DNS point bifurcates into two, a phenomenon not previously observed in applied models of this sort.

\section{The Model}

Consider an individual who holds a position vis a vis an organization. The organization is abstracted as a all of unit radius where distance from the origin measures the number of connections ("ties") the individual maintains with people outside of the organization relative to ties to people within the organization. So the origin represents being fully inward looking, immersed in one's own organization. The effort-minimizing path is for individuals within the organization to interact with and build relationships with others within the organization and for people outside the organization to interact with and build relationships with others outside the organization. So, in the absence of conscious effort, people outside the organization will tend to lose touch with what the organization is doing and people inside the organization will become more and more inwardly focused, (cf. Ref. DeGroot, 1974).

In such circumstances, organizations can become too incestuous, recycling ideas that were "invented here" and overlooking developments in the wider world. So it can be valuable for some people to stand at the "edge" of the or- 
ganization, connected to it but also strategically positioned as a bridge between the organization and the outside world.

For simplicity, assume the individual optimizing his or her position vis a vis the organization does so along a single dimension $x$. Generalizations to multiple dimensions would be of interest, but even this one-dimensional case proves insightful. Let the origin denote the "center" of the organization on this dimension and -1 and 1 denote its boundaries. As a further simplification, we will consider here a case in which the organization is symmetric about its center of gravity, but asymmetric cases could also be considered.

Our model of the natural evolution of social interaction is that the boundary is unstable. People within the organization gravitate toward its center. Those outside it are drawn toward other organizations and activities. Thus, the uncontrolled state dynamics might be take a form such as:

$$
\dot{x}=x^{3}-x,
$$

so $\dot{x}=0$ at both boundaries, the state converges toward the origin for $|x|<1$, and it diverges for $|x|>1$.

The individual can modify this trajectory in either direction by exerting some effort, denoted by the control variable $\nu$. It is conventional to assume that costs are a convex function of effort, and we will assume a quadratic dependence for simplicity. Ideally the individual would like to stand on the boundary between the organization and the outside world. We presume no distinctive benefit to being on the left-hand boundary vs. the right-hand boundary, but do assume that it is better for an individual to be a little too "close" to his or her own 
organization than a little too far. That is, we are imagining a situation in which the individual is an "employee" or otherwise receives compensation from the organization that is centered at the origin, so the individual is better off being "inside" the organization's boundary rather than a similar distance outside the boundary. Perhaps the simplest cost function satisfying these considerations is $\left(x^{2}-1\right)^{2}$, so our overall optimization problem becomes

$$
\begin{array}{r}
\min _{\nu} \int_{0}^{\infty} \exp (-r t)\left(\left(x^{2}-1\right)^{2}+c \nu^{2}\right) d t \\
\text { s.t. } \quad \dot{x}=x^{3}-x+\nu
\end{array}
$$

where $r$ is the individual's discount rate, and $c$ is a positive constant reflecting the cost of adjusting one's position.

\section{Analysis of the Model}

To solve this problem by applying Pontryagin's minimum principle (see, e.g., Refs. Feichtinger and Hartl, 1986; Leonard and Long, 1992) we consider the current value Hamiltonian

$$
H=\left(x^{2}-1\right)^{2}+c \nu^{2}+\lambda\left(x^{3}-x+\nu\right),
$$

where $\lambda$ denotes the co-state variable in current value terms.

Following the standard methods of optimal control theory we derive the necessary optimality condition

$$
\nu^{\star}=\arg \min _{\nu} H
$$


which implies

$$
\lambda=-2 c \nu \Leftrightarrow \nu=-\lambda / 2 c,
$$

by setting $H_{\nu}=0$.

Since the Legendre-Clebsch condition $H_{\nu \nu}=2 c>0$ is satisfied the continuity and uniqueness of the minimizing control $\nu^{*}$ along an optimal path is assured. Furthermore, the co-state equation is given by

$$
\dot{\lambda}=r \lambda-H_{x}=\lambda\left(1-3 x^{2}+r\right)-4 x\left(x^{2}-1\right) .
$$

Considering Eq. 4 we derive the differential equation for the control variable $\nu$

$$
\dot{\nu}=\nu\left(1-3 x^{2}+r\right)+2 x / c\left(x^{2}-1\right) .
$$

The state equation Eq. 1 and the adjoint equation Eq. 5, where $\nu$ is given by the Hamiltonian minimizing condition Eq. 4, yield the canonical system as necessary optimality conditions for our control problem.

\subsection{Regions of Stability Properties and Bifurcations}

We next examine the steady states of the canonical system as functions of its two parameters $r$ and $c$ and determine their stability properties. As we can see in Fig. 1, the parameter space is divided into five main regions, with different numbers of steady states and different stability properties. (See also Tab. 1.) The exact computations of the steady states and their properties for these regions can be found in Appendices A and B, respectively. The origin and the boundary states \pm 1 are always steady states. The steady states at \pm 1 are referred to as boundary steady states. One additional pair of steady states can 
emerge. These additional steady states are between the origin and the boundary steady states when the boundary steady states are saddles; otherwise they are outside the boundary steady states.

These five regions are divided by bifurcation lines. (See Fig. 1.) Crossing these curves can mean a change in the system's dynamic or optimal behavior. New equilibria can emerge while others disappear or change their stability properties. As the possibility of such limiting cases is zero, they are of no vital importance for applications, but they nevertheless give insight into the mathematical formulations of radical changes in the model behavior as parameters vary. While finding explicit formulae for the bifurcation lines was not surprising it was furthermore also possible to numerically calculate the heteroclinic bifurcation line $\mu$. Whereas changes in the model's dynamic behavior can take place at the other bifurcation lines, a change in the model's optimal behavior is given at the continuous policy line $\zeta$ and the heteroclinic bifurcation line $\mu$.

\section{Region I}

For parameters lying in Region I the only steady states are at the origin and at the boundaries \pm 1 . While the origin is an unstable focus the boundaries are saddles, and the region is delimited by the positive $r$ and $c$ axes and the curve (labeled $\alpha$ ) defined by $c=8 /(r+2)^{2}$. 


\section{Region II}

The only difference between Region I and Region II is the nature of the steady state at the origin, which in this case is an unstable node. As can be expected, there is an intermediate state of the origin at line $\alpha$, where the origin becomes a degenerate node.

Region II is bounded by the line $\alpha$ and bifurcation line $\beta$, defined by $c=$ $2 /(r+1)$.

\section{Region III}

Region III has five equilibria. The unstable node at the origin trifurcates into a saddle at the origin and two unstable nodes, while the boundary equilibria remain saddles. Moving from Region II to Region III, bifurcation line $\beta$ has to be crossed. As the origin trifurcates into a saddle and two unstable nodes we have the important case of a pitchfork bifurcation. As an intermediate state the origin becomes a degenerate fixed point. Its importance is indicated by the change in the optimal solution strategy as will be investigated in the next section.

Region III lies between the curves $\beta, \delta$ and $\gamma$, where the exact formulae for the latter is derived in Appendix B.

\section{Region IV}

Moving to Region IV the equilibria lying between the origin and the boundary equilibria, mutate from unstable nodes (Region III) to unstable foci. The prop- 
erties of the other equilibria remain unchanged. The bifurcation line $\gamma$ forms the boundary of this region, and the inner equilibria become degenerate nodes when crossing this curve.

\section{Region V}

While the saddle at the origin does not change in Region $\mathrm{V}$, the boundary equilibria become unstable nodes and the other pair of equlibria, now outside the boundary, change from unstable nodes to saddles, which is called a transcritical bifurcation. The curve labeled $\delta$ defined by $c=2 /(r-2)$ delimits Region V.

At curve $\delta$ a substantial change in the boundary equilibria takes place. Below this curve they were saddles. As parameter $c$ increases, approaching $\delta$ from below, the equilibria lying inside the boundary approach the boundary equilibria, until they collide with the outer equilibria at the bifurcation line $\delta$. The collision of the equilibria produces degenerate fixed points at the boundary. Moving on into Region V leaves unstable nodes behind at the boundary, whereas the former inner equilibria lie now outside the boundary and become saddles. This change has a considerable effect on the optimal solution. (See 3.2).

\section{Bifurcation line $\mu$ and line $\zeta$}

While the bifurcation lines mentioned so far separate regions with different properties and/or number of equilibria the heteroclinic bifurcation line $\mu$ and continuous policy line $\zeta$ lie inside such regions. Where the term continuous policy line denotes the fact that crossing this curve the optimal control at the 
DNS point becomes (dis)continuous. Both of them have been computed numerically, considering the behavior of the corresponding stable manifolds. These calculations give rise to the conjunction a heteroclinic bifurcation occurs, where two equilibria previously not connected by any orbit are now connected by a so called heteroclinic orbit (see cf. Ref. Guckenheimer and Holmes, 1983). Investigating such heteroclinic bifurcations is very interesting as they may give rise to DNS points (see Ref. Wagener, 2003), which is the case for our model. Crossing bifurcation line $\mu$ produces a dramatic change for the global optimal policy. Line $\zeta$ separates regions with only a slightly different local optimal behavior, viz the continuity of the optimal policy at the occuring unstable nodes. In contrast, when crossing the part of $\zeta$ lying in Region II, the optimal behavior at the origin changes, while for the part lying in Region III the inner equilibria are affected.

\subsection{Optimal Strategies}

Having analyzed the dynamic systems in terms of equilibria and their properties, we next explore when various strategies are optimal. It turns out that there are essentially three strategies that may be optimal depending on the values of parameters $r$ and $c$ : (A) move to the boundary $( \pm 1)$, (B) (almost) always fall off from the boundary to the origin or a state outside the boundaries, and (C) move either to the origin or the boundaries depending on one's initial position.

Strictly speaking it is not possible to give a theoretical argument that the extremals satisfying the necessary optimal conditions are really the unique opti- 
mal solutions of the problem. The convexity of the Hamiltonian in regard of the control $\nu$ alone is not strong enough to ensure global optimality of the extremal solution.

Nevertheless for the occurring saddles it can analytically be shown, via the Riccati differential equation (Ref. Maurer and Pickenhain, 1995), that they are at least locally optimal. The same holds true for the saddlepaths, whereas in this case the local optimality can only be shown numerically.

Summing up there is strong evidence for the global optimality of the extremals and it is consistent within the models interpretation. But as strong evidence is no mathematical category one should, strictly speaking, only classify the optimal solutions as extremals. When we use the term optimal solution after all, we do that for easier notational reasons and within the restrictions I mentioned before. Hence we can speak about the stability of the optimal solution, which are assumed to coincide with the calculated extremals.

The stability regions and bifurcation lines play an important role in defining when the various strategies are optimal. Bifurcation line $\mu$ in particular separates regions with different optimal behavior but the same dynamic behavior. In particular $\mu$ separates stability Region IV into two subregions, with that falling on the left of $\mu$ denoted IVa, respectively, and that to the right denoted IVb. In addition Region III is subdivided into three subregions by $\mu$ and $\zeta$, with Region IIIa to the left of line $\mu$, Region IIIb between $\mu$ and line $\zeta$, and Region IIIc to the right of $\zeta$.(See Appendix B for details.) So different strategies are optimal in different parts of a single stability region (namely Regions II, III and IV), and 
the same strategy may be optimal for different stability regions (e.g., Regions I, IIa, IIIa and IVa).

\section{Strategy A: Always Move to the Boundary}

In stability Regions I, IIa, IIIa and IVa, it is always optimal to move to one of the boundary states \pm 1 , depending only on the sign of the initial starting position $x_{0}$. That is for $x_{0}>0$ it is optimal to tend to state 1 , while state -1 is the long run optimal state for $x_{0}<0$. Only in the case when starting exactly at $x_{0}=0$ both options, moving to the left or right boundary, are equally optimal, whereas staying at the origin would be more expensive and hence be suboptimal. Therefore the origin is a so called DNS point (cf. Ref. Tragler et al., 2001). Note that an infinitesimally small deviation from the starting position $x_{0}=0$ leads to a finite change in the optimal initial level of effort $\nu_{0}^{*}$, that is to say the optimal policy is discontinuous at $x_{0}=0$.

Moving to the boundaries makes intuitive sense because in these regions parameters $r$ and/or $c$ are small. Clearly the boundary points are the most advantageous points. If the discount rate is small (decision maker is far sighted) and/or the cost of adjusting one's position is low enough, it is always worth investing the effort needed to reach one of these advantageous points.

Models with parameters $r$ and $c$ lying either exactly at $\mu$ or in Region IIb show slightly different optimal behavior at $x_{0}=0$. Moving to the boundary continues to be optimal for starting positions $x_{0} \neq 0$, but if $x_{0}=0$ it is optimal to remain at the origin. Moreover, deviating an infinitesimal distance from a 
starting position at $x_{0}=0$ leads only to an infinitesimally small change in the optimal initial level of effort $v_{0}^{*}$. That is, the optimal policy is continuous at $x_{0}=0$. This stands in contrast to the strategy described before, where we observed a discontinuous optimal policy. The continuity of optimal policy means moving to one of the boundaries optimally from $x_{0} \approx 0$ involves only an infinitesimally small exertion of effort and the cost for such a policy differs only infinitesimally from that of staying at the origin. Furthermore, the starting position $x_{0}=0$ is no longer a DNS point. But as the optimal policy left and right of $x_{0}$ differs we denote such a point of continuous optimal policy DNS threshold.

\section{Strategy B: (Almost) Always Fall Off from the Boundary}

Stability Region V represents the opposite case to strategy A. If the starting position is exactly at the boundary, it is optimal to stay there. Otherwise, parameters $r$ and $c$ are large enough that if the decision maker deviates even from the boundary, the decision maker is short-sighted to such a degree and the costs of control are so high that the benefits of returning to the boundary are not worth the effort. So for starting positions inside the boundary $\left(\left|x_{0}\right|<1\right)$ it is optimal to tend to the origin, while for starting positions outside the boundary it is optimal to move to the equilibria outside the boundaries. The optimal policy at the boundary equilibria is continuous and therefore starting exactly at this equilibria means staying put. Therefore the long run optimal behavior is sensitive to the exact initial starting position near the boundary equilibria. 


\section{Strategy C: Move to the Origin if One Starts Nearby; Otherwise move}

\section{to the boundary}

Regions IIIb and IVb present an intermediate case to some extent. If the decision maker's initial position is inside the boundary and close to the origin, then it is optimal to move to the origin. But if the initial position is inside the boundaries $\left(\left|x_{0}\right|<1\right)$ but farther from the origin, it is optimal to move out to a boundary, specifically the closer one. In between there are points of indifference, one on either side of the origin, from which the decision maker is equally happy moving left or right. Note that the optimal policy is discontinuous at these points of indifference (especially for Region IIIb, where the inner equilibria are unstable nodes). Therefore these points of indifference are DNS points. If one starts outside the boundaries returning to the boundaries is always worth the effort, presumably because of the heavy penalty in the objective function for being far from the boundary, and the cubic term in the state dynamics that tends to drive states that are outside the boundary further away from the boundary at an ever increasing rate.

Models with parameters $r$ and $c$ lying in Region IIIc show a slightly different optimal behavior at the inner equilibria (unstable nodes). In contrast to the strategy considered before the optimal policy is continuous at these equilibria. Hence staying at these points become optimal and they are no longer DNS points but DNS thresholds. 


\subsection{Change in Optimal Strategy As Parameters Vary}

This section examines in more detail how the optimal strategy varies as one of the two parameters in turn is increased.

\section{Increasing $r$ for Fixed Values of the Cost Parameter $c$}

Figure 2 shows how the optimal solution changes for a given cost parameter $c$ as the discount rate parameter $r$ increases, i.e., as the decision maker gets more and more myopic. In particular, Figures 2a-d show phase portraits when $c=2.5$ and the discount rate parameter $r$ is $0.2,0.4,2.5$ and 3.5 respectively.

When $c=2.5$ and $r$ is small it is always optimal to converge to a boundary, specifically the closer one. If one starts at the origin moving left or right generates the same costs while remaining at the origin is more expensive. Therefore a decision has to be made arbitrarily between moving to the left or right boundary. (See Fig. 2a). This statement holds true for every parameter $r$ and $c$ lying below bifurcation lines $\alpha$ or $\mu$. Increasing $r$ leads the stable manifolds of the boundary equilibria at the $\nu$-axis to approach the origin, until the origin lies precisely on the stable manifolds for $r=1 / 2.5$. This is the case of a heteroclinic orbit. For this hairline case the origin becomes a point with continuous optimal policy at $x_{0}=0$. (See Fig. $2 \mathrm{~b}$ ). If $r$ increases further the DNS point at the origin bifurcates into two DNS points. Starting between these points moving to the origin is optimal, while converging to the boundary is optimal for initial starting positions outside the DNS points. This optimal behavior does not change upon crossing bifurcation line $\gamma$, however the equilibria inside the boundaries 
change from unstable foci to unstable nodes and the inner equilibria become points with a continuous policy function. (See Figs. 2c and 3d) I.e. to the left of $\mu$ (in Region IVb) a decision maker - starting at the inner equilibria - should choose (arbitrarily) to move left or right. To the right of $\mu$ (in Region IIIc) a decision maker should stay put. Letting $r$ grow further, the equilibria inside the boundaries move towards the boundary equilibria. Reaching bifurcation line $\delta$ these equilibria coincide leaving degenerate fixed points behind at \pm 1 . At this limiting case the optimal behavior can only be analyzed with standard methods for initial starting positions inside the boundaries, where the optimal paths converge to the boundary equilibria. For discounting rates $r$ above bifurcation line $\delta$ the optimal behavior changes dramatically. As the boundary equilibria become unstable nodes, it is never optimal to converge to these equilibria. Instead the origin becomes optimal for every state starting inside the boundary, while outside the boundaries the optimal solution paths converge to equilibria with absolute state values greater than 1. (See Fig. 2d.)

For $c<1$ two other cases can occur as $r$ increases. Letting e.g. $c=0.8$, a small $r$ implies optimal paths converging to the boundaries and $x_{0}=0$ becoming a DNS point where the costs for moving to the left or to the right equilibria are the same. Crossing bifurcation line $\alpha$ the optimal behavior at the origin remains unchanged until bifurcation line $\zeta$ is reached. As long as we are in Region II there is no change in the global optimal behavior as described in the Sec. 3.1 for bifurcation line $\zeta$. But for starting positions at $x_{0}=0$ the possible optimal solutions change. Below bifurcation line $\zeta$ it is optimal to exert a finite 
initial effort $\nu_{0}^{\star}$ to move to the boundary. That means if the decision maker is farsighted enough he or she accepts a higher initial effort and moves to one of the boundaries. Getting more myopic the decision maker no longer invests in a high initial effort, and staying at the origin becomes optimal. This is the case when the Policy function at the origin becomes continuous as described in the Sec. 3.2.

Increasing $r$ further and crossing bifurcation line $\beta$ the unstable node at the origin trifurcates into two unstable nodes at the inner equilibria and a saddle at the origin. For our choice of $c$ we get a continuous policy function at the unstable nodes. Therefore if one starts near the origin, one returns to the origin. For all initial starting positions outside the unstable nodes moving to the boundary equilibria is worth the effort. While starting exactly at the unstable nodes, means staying put.

\section{Increasing $c$ for Fixed Values of the Discount Rate $r$}

Figure 3 shows phase portraits when $r=0.8$ and the cost parameter $c$ is $0.5,1.05,1.15$, and 2.5 , respectively. When costs are low $(c=0.5$; Fig. 3a) starting left (right) from the origin it is optimal to move to the left (right) boundary, while if starting at $x_{0}=0$ the decision maker can choose arbitrarily between moving to the left or right boundary. As $c$ increases further towards the bifurcation line $\zeta$, the initial level of effort $\nu_{0}^{\star}$ for starting positions deviating only slightly from $x_{0}=0$, shrinks to 0 , while the optimal behavior remains the same as before. This is clear from the decision maker's point of view as the 
effort is getting more and more costly. (See Fig. 3b.) Crossing $\mu$ the stable manifolds coincide at the origin and the difference in the initial level effort is 0 . Therefore the optimal policy becomes continuous at the origin and we achieve the hairlinecase where staying at the origin becomes optimal. Crossing $\mu$ the region where moving towards the origin is optimal expands and two DNS points appear. To the left (right) of the left (right) DNS point it is optimal to converge to the left (right) equilibrium, while between the DNS points moving to the origin becomes optimal. Now the costs are so high that it is not worth moving to the boundaries when starting near the origin. (See Fig. 3d.)

The cases described before characterize the behavior for all models with $r \leq 1$, while two slight differences take place for $r>1$, as can be explained considering Fig. 1. Whereas $\mu$ make up the limiting case for the emergence of two DNS points for $r \leq 1$ the continuation of $\mu$ (part of $\zeta$ lying in region II) only divides regions with different local optimal behavior at the origin for $r>1$ as described in the Sec. 3.1. Increasing the $\operatorname{costs} c$ leads to a shrinking gap between the initial level effort $\nu_{0}^{\star}$ for starting positions on either side of $x_{0}=0$. Nevertheless costs are low enough to be worth the effort of moving to one of the boundaries, even if one starts at $x_{0}=0$. As the costs get higher (above $\zeta$ ) moving to the closer boundary is only infinitesimally more expensive then staying at the origin and therefor staying at the origin becomes optimal. If one starts near the origin it is optimal to stay near the origin for a while and not move away too quickly. The higher the costs are increasing the longer the duration for staying near the origin. Crossing bifurcation line $\beta$ the movement 
near the origin comes to a stillstand. This is the case where the origin becomes a degenerate fixed point. Raising the costs above the bifurcation line $\beta$ the movement near the origin is reversed and moving to the origin becomes optimal when starting nearby. As a consequence, two starting positions $x_{0}$ become points of indifference between moving to the origin and moving to the boundary. Augmenting the costs further moves these points of indifference out toward the outer equilibria. But for $r \leq 2$ the outer equilibria still remain optimal at least for starting positions with $\left|x_{0}\right| \geq 1$ and in a shrinking neighborhood inside the boundaries. If $r>2$ the situation changes and crossing bifurcation line $\delta$ the boundary equilibria are only optimal in the case of starting exactly at \pm 1 . For every other case the origin is optimal, starting inside the boundaries $\left|x_{0}\right|<1$, and equilibria outside the boundaries become optimal for starting positions meeting $\left|x_{0}\right|>1$.

\section{Conclusion}

In this model, positioning oneself as a bridge between one's own organization and the outside world yields benefits but also takes effort. Whether moving to a bridging position is worthwhile depends on how costly it is to alter one's position and on how far sighted one is. Individuals who are sufficiently myopic and for whom such movement is sufficiently painful should not bother. Those who are sufficiently far-sighted and/or flexible should always become bridges. For others, the optimal strategy depends on whether one is initially close to or 
far from being such an organizational bridge.

Because of the relative simplicity of this model, the model's structure and resulting optimal behavior could be fully characterized in the parameter space. In particular, it was possible to find explicit solutions for every bifurcation line, including the heteroclinic bifurcation at the origin. Furthermore the lines where the optimal policy becomes continuous at the relevant unstable nodes were numerically calculated.

This solution yielded quite a number of mathematically interesting structures. Even though it is a one state model, varying a single parameter generates instances of zero, one, or two DNS points and even instances in which a single DNS point trifurcates into two DNS point and a saddle point. More generally we found regions with the same number and properties of equilibria but different optimal behavior, divided by a heteroclinic bifurcation line, and regions where the optimal solution was sensitive to the exact starting position

This simple model may have interesting extensions. One would replace the one-dimensional (one-state) model of the organization with a two-dimensional or even n-dimensional model. The unit circle or unit sphere, respectively, could still denote the boundaries of the organization. The state dynamics could still be taken such that people within the organization gravitate toward its center at the origin whereas those outside the boundary are drawn further away. And the cost function could still reflect the ideal of staying at the boundary or some selected points along that boundary. Considering such a model, one could expect to find two-dimensional DNS curves and a DNS point at the origin with an arbitrarily 
large number of alternative optimal strategies available.

Another variation would recognize that people can be members of more than one organization simultaneously, so the objective function could be the sum of distances from the centers of several different organizations. Again, in a two- or higher-dimensional model, this might likewise yield rather complex and interesting solutions.

\section{A Existence of equilibria}

Considering the dynamical system

$$
\begin{aligned}
\dot{x} & =x^{3}-x+\nu \\
\dot{\nu} & =\nu\left(1-3 x^{2}+r\right)+2 x / c\left(x^{2}-1\right) .
\end{aligned}
$$

the equilibria must satisfy

$$
\begin{aligned}
& \nu=x-x^{3} \\
& \nu=2 x\left(1-x^{2}\right) /\left(c\left(1-3 x^{2}+r\right)\right) .
\end{aligned}
$$

Setting these expressions for $\nu$ equal to each other we get the following solutions for $x$ :

$$
\begin{aligned}
x_{1} & =0 \\
x_{2,3} & = \pm 1 \\
x_{4,5} & = \pm \sqrt{(1+r-2 / c) / 3},
\end{aligned}
$$


and therefore abbreviating $w=\sqrt{(1+r-2 / c) / 3}$ the formal solutions of the fixpoints are

\begin{tabular}{c|c|c} 
& $\hat{x}$ & $\hat{\nu}$ \\
\hline$E_{1}$ & 0 & 0 \\
$E_{2}$ & 1 & 0 \\
$E_{3}$ & -1 & 0 \\
$E_{4}$ & $w$ & $w\left(1-w^{2}\right)$ \\
$E_{5}$ & $-w$ & $-w\left(1-w^{2}\right)$.
\end{tabular}

Having in mind the interpretation of -1 and 1 as the boundary states, we refer to $E_{2}$ and $E_{3}$ as the boundary equilibria. While $E_{1}-E_{3}$ are global solutions for Eq. 8, we have to determine the regions of existence for the equilibria $E_{4}$ and $E_{5}$. As they only depend on $w$ we have to consider the case where $w$ is real, implying

$$
\begin{aligned}
1+r-2 / c & \geq 0 \\
c & \geq 2 /(r+1) .
\end{aligned}
$$

That is for $c$ sufficing Eq. 8 the equilibria $E_{4}$ and $E_{5}$ exist.

At least we determine the cases where these equilibria coincide with the other equilibria.

Case 1: $w=0 \Leftrightarrow c=2 /(r+1)$ the equilibria coincide with the origin.

Case 2: $w=1 \Leftrightarrow c=2 /(r-2) E_{2}, E_{4}$ and $E_{3}, E_{5}$ respectively coincide. Beneath this curve the value of the state variable of $E_{4}$ and $E_{5}$ are smaller 
than 1 , while above this curve the equilibria are lying outside the boundary equilibria.

These results are summarized in Tab. 1.

\section{B Stability properties}

Knowing the number of equilibria for the different regions, we analyze now their stability properties. The characterization of the equilibrium behavior ensues from calculating the determinant, trace and discriminant of the Jacobi matrix $J$.

We get the common form of $J$, by linearizing the system of differential equations Eq. 7

$$
J(x, \nu)=\left(\begin{array}{cc}
3 x^{2}-1 & 1 \\
-6 x \nu+2 / c\left(3 x^{2}-1\right) & 1-3 x^{2}+r
\end{array}\right)
$$

calculating $\Delta, \tau$ and $D$ gives

$$
\tau=r
$$

$\Delta=\left(3 x^{2}-1\right)\left(1-3 x^{2}+r-2 / c\right)+6 x \nu$

$$
D=r^{2}-4\left(\left(3 x^{2}-1\right)\left(1-3 x^{2}+r-2 / c\right)+6 x \nu\right)
$$

with

$$
\begin{array}{ccc}
\tau & \ldots & \operatorname{tr}(J) \\
\Delta & \ldots & \operatorname{det}(J) \\
D & \ldots & \tau^{2}-4 \Delta .
\end{array}
$$


In the following subsections these formal results will be analyzed for the different equilibria.

\section{Origin}

At the origin the Jacobi matrix Eq. 9 simplifies to

$$
J(0,0)=\left(\begin{array}{cc}
-1 & 1 \\
-2 / c & r+1
\end{array}\right),
$$

and so we get

$$
\begin{aligned}
\tau & =r \\
\Delta & =-r-1+2 / c \\
D & =r^{2}+4 r+4-8 / c
\end{aligned}
$$

The stability properties are completely determined by the signs of the three parameters $\Delta, \tau$ and $D$. As $\tau=r>0$ always holds we only have to consider the occurrence of $\Delta=0$ and $D=0$. Solving these equations we get

$$
\begin{aligned}
& \Delta=0 \quad \Leftrightarrow \quad c=2 /(r+1) \\
& D=0 \quad \Leftrightarrow \quad c=8 /(r+2)^{2}
\end{aligned}
$$

Distinguishing the cases where the sign of $\Delta$ and $D$ change we get five regions in parameter space.

Case 1: $c<8 /(r+2)^{2} \Rightarrow \Delta>0 \wedge D<0$ characterizes an unstable spiral.

Case 2: $c=8 /(r+2)^{2} \Rightarrow \Delta>0 \wedge D=0$ gives the limiting case of a degenerated node at the origin (labeled as $\alpha$ ). 
Case 3: $8 /(r+2)^{2}<c<2 /(r+1) \Rightarrow \Delta>0 \wedge D>0$ is associated with an unstable node.

Case 4: $c=2 /(r+1) \Rightarrow \Delta=0 \wedge D>0$ implies the critical case of a degenerate fixed point (labeled as $\beta$ ).

Case 5: While for $c>2 /(r+1) \Rightarrow \Delta<0 \wedge D>0$ the origin is a saddle.

\section{Boundary Equilibria}

In case of a boundary equilibria the Jacobi matrix Eq. 9 becomes

$$
J=\left(\begin{array}{cc}
2 & 1 \\
4 / c & r-2
\end{array}\right)
$$

and

$$
\begin{aligned}
\tau & =r \\
\Delta & =2 r-4-4 / c \\
D & =r^{2}-8 r+16+16 / c .
\end{aligned}
$$

As Eq. 14 has no real root for $c>0, D$ does not change sign and hence $D>0$ in the whole parameter space. Therefore only the regions where $\operatorname{sgn}(\Delta)$ differs have to be considered.

But $\Delta=0 \Leftrightarrow c=2 /(r+1)$, so we can distinguish three different regions

Case 1: $c<2 /(r+2) \Leftrightarrow \Delta<0$ characterizes a saddle.

Case 2: $c=2 /(r+2) \Leftrightarrow \Delta=0$ gives the critical case of degenerate fixed points.

Case 3: $c>2 /(r+2) \Leftrightarrow \Delta>0$ is associated with an unstable node. 


\section{Equilibria $E_{4}$ and $E_{5}$}

In case of equilibria $E_{4}$ and $E_{5}$ the Jacobi matrix Eq. 9 becomes

$$
J=\left(\begin{array}{cc}
3 w^{2}-1 & 1 \\
-6 w^{2}\left(1-w^{2}\right)+2 / c\left(3 w^{2}-1\right) & r-3 w^{2}+1
\end{array}\right)
$$

and

$$
\begin{aligned}
\tau & =r \\
\Delta & =\left(3 w^{2}-1\right)\left(1-3 w^{2}+r-2 / c\right)+6 w^{2}\left(1-w^{2}\right) \\
D & =r^{2}-4\left(3 w^{2}-1\right)\left(1-3 w^{2}+r-2 / c\right)+24 w^{2}\left(1-w^{2}\right) .
\end{aligned}
$$

Resubstituting $w$ in Eq. 15 the factor $1-3 w^{2}+r-2 / c=0$ and therefore $\Delta$ and $D$ reduce to

$$
\begin{aligned}
& \Delta=6 w^{2}\left(1-w^{2}\right) \\
& D=r^{2}-24 w^{2}\left(1-w^{2}\right)
\end{aligned}
$$

Setting $\Delta=0$ we find the solutions

$$
\Delta=0 \Leftrightarrow\left\{\begin{array}{lc}
c=2 /(r+1) & \forall r \\
c=2 /(r+1) & r>2 .
\end{array}\right.
$$

Finding the solutions for $D=0$ is straight forward.

$$
\begin{aligned}
D=0 & \Leftrightarrow \quad r^{2}-24 w^{2}\left(1-w^{2}\right)=0 \\
& \Leftrightarrow \quad w^{4}-w^{2}+r^{2} / 24=0 \\
& \Leftrightarrow \quad w^{2}=1 / 2 \pm \sqrt{\left(6-r^{2}\right) / 24} \\
& \Leftrightarrow \quad(r+1-2 / c) / 3=1 / 2 \pm \sqrt{\left(6-r^{2}\right) / 24}
\end{aligned}
$$


For notational simplicity we set $k=2 / c$ and get

$$
\begin{aligned}
2 r-1-2 k & = \pm 6 \sqrt{\left(6-r^{2}\right) / 24} \\
(2 r-1-2 k)^{2} & =3 / 2\left(6-r^{2}\right) \\
k^{2}+k(1-2 r) & =-\left(11 r^{2}-8 r-16\right) / 8
\end{aligned}
$$

a quadratic in $k$ and after resubstituting $2 / c$ for $k$ we find the solutions

$$
c_{1,2}=4\left(4 r-2 \pm \sqrt{6\left(6-r^{2}\right)}\right) /\left(11 r^{2}-8 r-16\right) .
$$

These solutions $c_{1}$ and $c_{2}$ form a curve in the parameter space which are continuously connected at $r=\sqrt{6}$. As the polynomial in the denominator of Eq. 16 has a positive real root at $r=4 / 11(1+2 \sqrt{3})$ the solutions have a singularity at this point, that can be lifted in the case of $c_{2}$, while it is a real singularity in the case of $c_{1}$.

Summarizing these considerations we can distinguish six different regions, for the properties of the equilibria.

Case 1: $c=2 /(1+r) \Rightarrow \Delta=0 \wedge D>0$ entails that $E_{4}$ and $E_{5}$ coincide with the origin and can be characterized as degenerate fixed points.

Case 2: $2 /(1+r)<c<2 /(r-2)$ and $c$ not inside region III then $\Delta>0 \wedge D>0$ implies that the equilibria are unstable nodes.

Case 3: $c \in \gamma$ gives the limiting case of a degenerated node.

Case 4: If $c$ lies in region III then $\Delta>0 \wedge D<0$ is associated to the case of unstable foci. 
Case 5: $c=2 /(r-2) \Rightarrow \Delta=0 \wedge D>0$ let the equilibria coincide with the boundary equilibria and have the properties of degenerate fixed points (labeled as $\delta)$.

Case 6: $c>2 /(r-2) \Rightarrow \Delta<0 \wedge D>0$ gives the case of saddles.

These results are summarized at Tab. 1.

\section{References}

KrackHARDT, D. , Assessing the political landscape: Structure, cognition, and power in organizations, Administrative Science Quarterly, 35:342-369, 1990.

Brass, D. J. And Burkhardt, M. E. , Centrality and Power in Organizations, pages 191-215, Harvard Business School Press, Boston, MA, 1992.

Freeman, L. C. , Centrality in social networks: Conceptual clarification, Social Networks, 1:215-239, 1979.

Granovetter, M. S. , The strength of weak ties, American Journal of Sociology, 78:1360-1380, 1973.

Burt, R. , The Social Structure of Competition, pages 57-91, Harvard Business School Press, Boston, MA, 1992.

Dechert, W. D. And Nishimura, K. , A complete characterization of optimal growth paths in an aggregated model with a non-concave production function, Journal of Economic Theory, 31:332-354, 1983.

SkIBA, A. K. , Optimal growth with a convex-concave production function, Econometrica, 46:527-539, 1978.

DeGroot, M. , Reaching a consensus, Journal of the American Statistical Association, 69:118-121, 1974.

Feichtinger, G. And HARTL, R. F. , Optimale Kontrolle ökonomischer Prozesse, Walter de Gruyter, 1986.

Leonard, D. And Long, N. V. , Optimal Control Theory and Static Optimization in Economics, Cambridge University Press, 1992.

GuCkenheimer, J. AND Holmes, P. , Nonlinear Oscillations, dynamical Systems and Bifurcations of Vector Fields, volume 42 of Applied mathematical sciences, Springer-Verlag, New York, 1983. 
Wagener, F. O. O., Skiba points and heteroclinic bifurcations, with applications to the shallow lake system, Journal of Economic Dynamics and Control, 27(9):1533-1561, July 2003.

Maurer, H. and Pickenhain, S., Second-order sufficient conditions for control problems with mixed control-state constraints, Journal of Optimization Theory and Applications, 86(3):649-667, 1995.

Tragler, G. , Caulkins, J. P. , and Feichtinger, G., Optimal dynamic allocation of treatment and enforcement in illicit drug control, Operations Research, 49(3):352-362, 2001. 


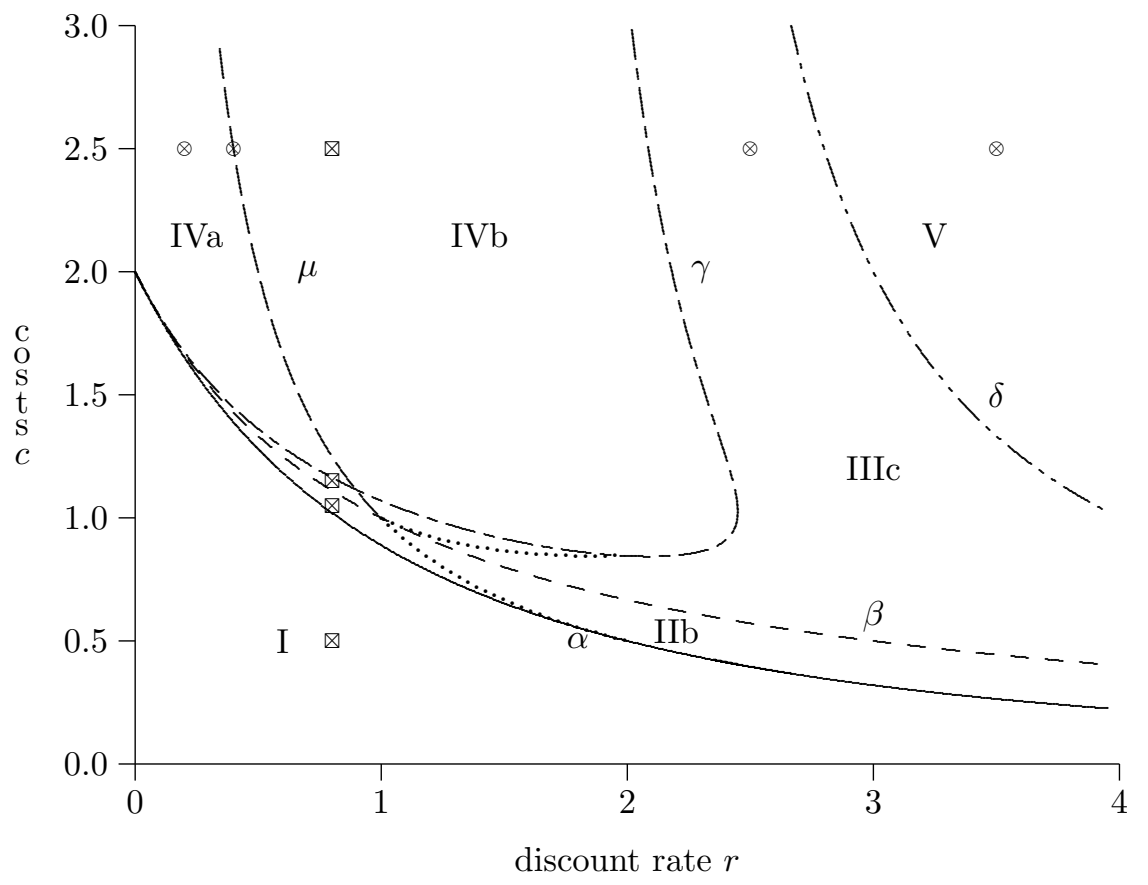

Figure 1: Regions of different stability and optimality divided by bifurcation lines $\alpha, \beta, \gamma, \delta$ the heteroclinic bifurcation line $\mu$ and line $\zeta$.

Region III is separated into three parts Region IIIa-IIIc by $\mu$ and the line $\zeta$. The line $\zeta$ in Region II, divides Region II into two parts Region IIa and Region IIb. To avoid cluttering the figure Regions IIa, IIIa and IIIb are not marked in Fig. 1.

$\otimes$ and $\otimes$ indicate different parameter sets for models depicted in Fig. 2 and Fig. 3. 


\section{Caption}

$-\alpha$ with $c=8 /(r+2)^{2}$

- - $\beta$ with $c=2 /(r+1)$

- - $\quad \gamma$ with $c=\left(4\left(4 r-2 \pm \sqrt{6\left(6-r^{2}\right)}\right)\right) /\left(11 r^{2}-8 r-16\right)$

$\ldots-\delta$ with $c=2 /(r-2)$

- - - $\mu$ numerically approximated

$\zeta$ numerically approximated

$\otimes \quad c=2.5$

$\bigotimes \quad r=0.8$ 

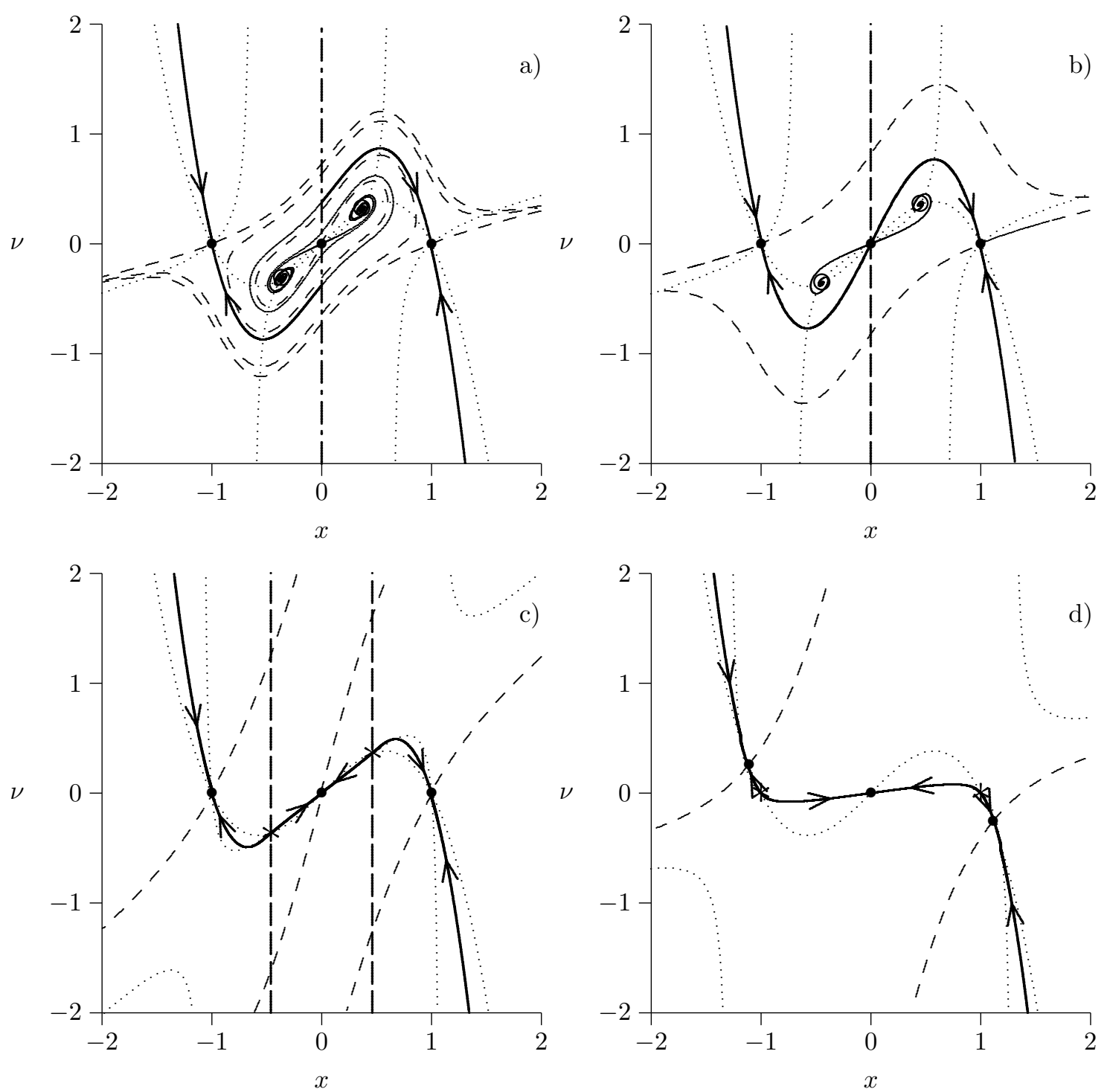

Figure 2: For constant cost $c=2.5$ and different discount rates $r$ the system dynamics is shown together with its optimal behavior and direction, starting in the upper left and moving clockwise the four cost parameters are

a) $r=0.2$ b) $r=0.4$ c) $r=2.5$ d) $r=3.5$ 


\section{Caption}

_ $\quad$ stable manifold

— optimal path

_ - unstable manifold

isoclines

-- DNS point

- - DNS threshold

- $\quad$ saddle

○ unstable focus

* unstable node

$\rightarrow$ direction of optimal path 

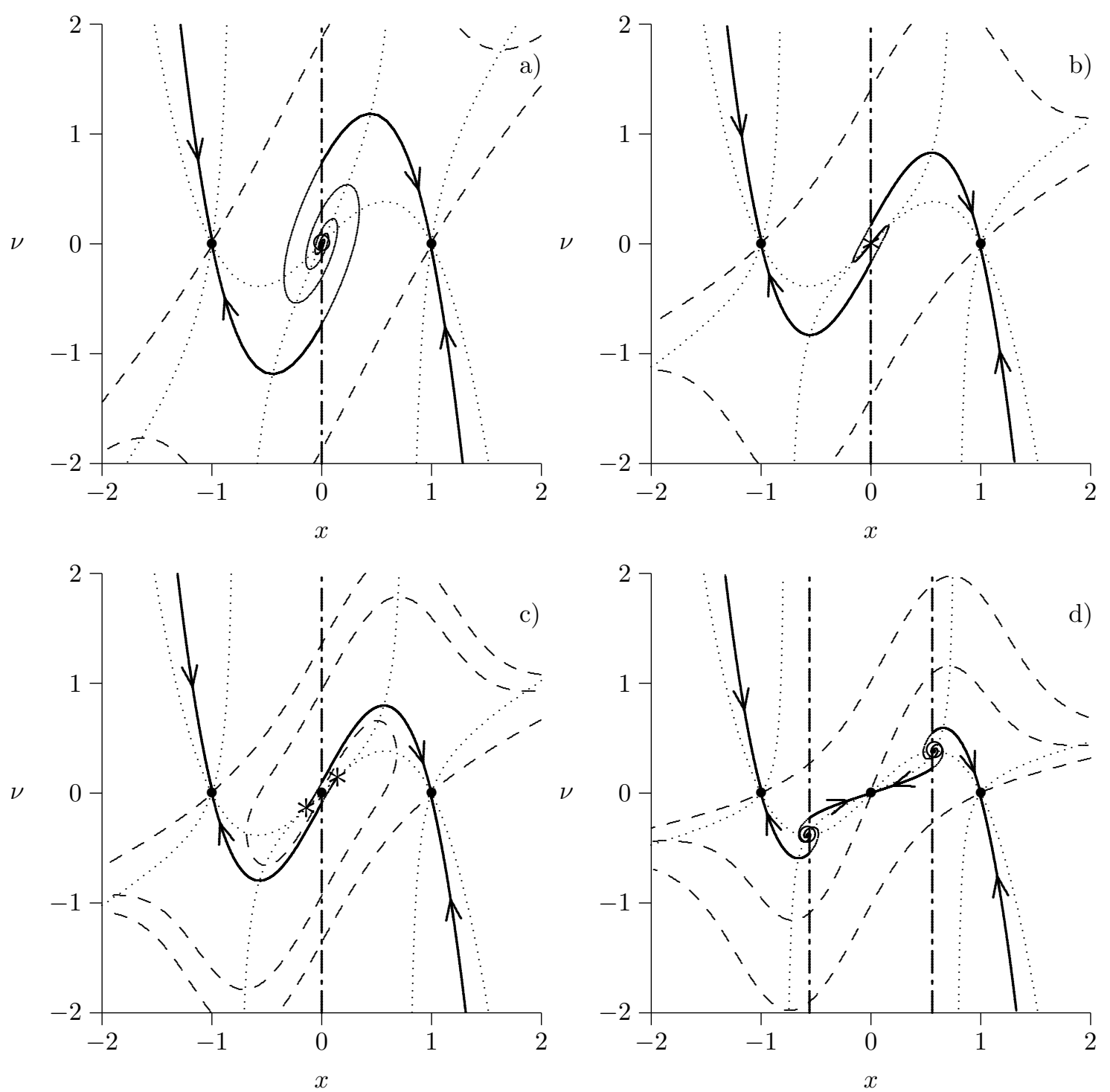

Figure 3: For constant discount rate $r=0.8$ and different costs $c$ the system dynamics is shown together with its optimal behavior and direction, starting in the upper left and moving clockwise the four cost parameters are

a) $c=0.5 \mathrm{~b}) c=1.05 \mathrm{c}) c=1.15 \mathrm{~d}) c=2.5$ 
Character of Equilibria at:

\begin{tabular}{|c|c|c|c|c|}
\hline Region/Curve & Origin & Boundary Equilibria & $E_{4}$ and $E_{5}$ & \# of DNS points \\
\hline I & unstable focus & saddle & - & 1 \\
\hline II a & unstable node & saddle & - & 1 \\
\hline II b & unstable node & saddle & - & - \\
\hline III a & saddle & saddle & unstable node & 1 \\
\hline III b & saddle & saddle & unstable node & 2 \\
\hline III c & saddle & saddle & unstable node & - \\
\hline IV a & saddle & saddle & unstable focus & 1 \\
\hline IV b & saddle & saddle & unstable focus & 2 \\
\hline $\mathrm{V}$ & saddle & unstable node & saddle & - \\
\hline
\end{tabular}

Table 1: Number and properties of equilibria. See Fig. 1 for definitions of regions and bifurcation curves. 